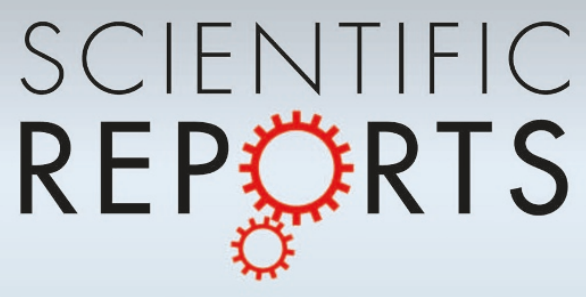

OPEN Absence of Predispositional Attentional

SUBJECT AREAS:

PERCEPTION

AMYGDALA

ATTENTION

AUTISM SPECTRUM DISORDERS

\title{
Sensitivity to Angry Faces in Children with Autism Spectrum Disorders
}

\author{
Tomoko Isomura', Hiroyasu Ito², Shino Ogawa ${ }^{3} \&$ Nobuo Masataka'
}

Received

${ }^{1}$ Primate Research Institute, Kyoto University, Inuyama, Japan, ${ }^{2}$ Research Institute of National Rehabilitation Center for Persons with

3 July 2014

Disabilities, Tokorozawa, Japan, ${ }^{3}$ Graduate School of Medicine, Kyoto University, Kyoto, Japan.

Accepted

28 November 2014

A rapid allocation of attention towards threatening stimuli in the environment is crucial for survival. Angry facial expressions act as threatening stimuli, and capture humans' attention more rapidly than emotionally positive facial expressions - a phenomenon known as the Anger Superiority Effect (ASE). Despite atypical emotional processing, adults with Autism Spectrum Disorders (ASD) have been reported to show ASE

Published

18 December 2014 similar to typically developed (TD) individuals. One important question is whether the basic process for ASE is intact in individuals with ASD or whether instead they acquire an alternative process that enables ASE. To address this question, we tested the prevalence of ASE in young children with and without ASD using a face-in-the-crowd task. ASE was clearly observed in TD children, whereas ASD children did not

Correspondence and requests for materials show the effect. In contrast to previous reports of ASE in adults or relatively older children with ASD, our results suggest that in ASD basic predispositional mechanisms to allocate attention quickly towards angry should be addressed to

T.I. (isomurat8818@ faces are not preserved.

gmail.com)

T he ability to understand others' emotional expressions plays an important role in our social interactions. In particular, the rapid detection of threatening social stimuli and modifying our behaviors according to the context is advantageous for avoiding social conflict. It is therefore thought that the human visual system may have evolved to be more sensitive to threatening faces than to other facial expressions ${ }^{1,2}$.

Angry faces are universally treated as signals of potential threat. They are processed rapidly and efficiently, and are particularly efficient in capturing attention ${ }^{3}$. This phenomenon is referred to as the Anger Superiority Effect (ASE). The neural networks underlying the facilitated processing of angry faces are less clear, but it has been suggested that re-entrant feedback loops from limbic regions (amygdala) to the visual cortex lead to amplified perceptual processing $g^{4-6}$.

ASE has been studied using a visual search paradigm that requires participants to search for a discrepant angry or happy face in a crowd of distractor faces (i.e., the Face-in-the-crowd task) ${ }^{7-9}$. Literally, ASE is defined as quicker and more accurate detection of an angry face than a happy face in a crowd of distractor faces. Recently, schematic face stimuli have frequently been used in studies demonstrating $\mathrm{ASE}^{1,10-12}$. By using schematic faces it is possible to eliminate many low-level perceptual variations found in photographs of emotional expressions, while allowing greater control of experimental variables.

ASE has recently been tested in participants with Autism Spectrum Disorders (ASD), using the visual search paradigm with schematic faces ${ }^{13}$, photographic faces ${ }^{14}$, and cartoon faces ${ }^{15}$. ASD are neurodevelopmental disorders characterized by social communicative difficulties and restricted behaviors and interests ${ }^{16}$. Previous studies have reported that individuals with ASD show specific difficulties in social and emotional information processing ${ }^{17}$. Dysfunction of the amygdala is thought to be one of the possible causes of ASD, possibly underlying atypical emotional responses in $\mathrm{ASD}^{18,19}$. In particular, recent studies revealed that individuals with ASD showed undifferentiated affective response to different facial emotions, assessed by event-related potentials (ERPs) ${ }^{20}$ and by facial electromyography (EMG) ${ }^{21}$.

However, in contrast to atypical emotional responses in ASD, studies have revealed that, similar to typically developing (TD) adults, adults with ASD also show ASE $^{13,14}$, although the effect appears less robust in the latter. Large or widely varying crowd sizes may impair performance in ASD to a greater extent than in TD individuals. These results suggest the possibility that individuals with ASD employ compensatory but less effective mechanisms that have been acquired later in development. To explore this possibility, it is necessary to examine ASE in younger participants. In one previous study 7- to 17-year-old children and adolescents with ASD aged showed 
ASE as clearly as TD individuals ${ }^{15}$. However, that study may have missed an initial atypicality in children with ASD due to the wide range of ages of the participants. Moreover, that study analyzed only accuracy data, although response times are more meaningful in the sense of adaptive mechanisms of "quick" allocation of attention. Therefore, in the present study, we focused on ASE in young children (7 to 10 years of age) to assess any initial atypicality of the effect by employing more valid methods, which included the analysis of response times and use of a child-friendly procedure. We hypothesized that even young TD children would clearly show ASE, as the rapid processing of angry faces is thought to be an evolutionarily preserved mechanism independent of age or experience ${ }^{1,22}$. By contrast, we made no prediction about how ASD children would perform on the same task.

We employed a visual search paradigm with schematic face stimuli, in which participants were required to detect a discrepant face as quickly as possible. In the current study, we used a touch-sensitive monitor to measure the response. Unlike many previous studies that used a keyboard or button for participants' resposes ${ }^{1,8,13,14,22,23}$, we used a recently developed touch-screen method that has also been reported to show a clear effect of threat detection advantage $\mathrm{e}^{2-26}$. In the keyboard or button procedure, participants are required to judge the presence or absence of a target within a matrix. In the touchscreen method, by contrast, participants are required to directly touch the target on the monitor, which makes the task more intuitive and easier for young participants. We additionally ran another type of visual search task in which simple lines were used as stimuli. This was done as a control task to explore group differences in processing of geometric features that were used to construct the schematic face stimuli, and in their performance of the visual search task. Previous studies have shown normal or even superior ability of visual search for non-social stimuli by people with ASD compared to TD individuals ${ }^{27-29}$. This superior performance was also observed in high autism quotient (AQ) individuals compared to low AQ individuals among non-clinical participants ${ }^{30,31}$. People with ASD appear generally good at single-target search due to their enhanced perception of stimulus features and discriminative ability. Although visual search in ASD has been well investigated, few studies have compared this population's performance with social versus non-social stimuli. In the present study, we first conducted two types of visual search task with both social and non-social stimuli using the same methods and same participants with ASD. This comparison allowed us to clarify whether their performance in the face-in-the-crowd task is specific to social stimuli or not. In the visual search task with social stimuli, the target was a schematic angry or happy face surrounded by a crowd of neutral faces. In the visual search of non-social stimuli, a tilted line was surrounded by a crowd of vertical lines, or vice versa.

\section{Results}

The median response time (RT) and relative accuracy (percentage of correct response over all trials) were calculated individually for each condition separately. RTs were analyzed only on correct trials. Mean accuracy and response times for each TD and ASD group in the two tasks are listed in Table 2. Although we analyzed both measures, the RTs were our main focus because the tasks were designed to produce very few errors. All statistical analysis was performed using SPSS 22 (IBM Japan, Ltd)

Face Task. A GLM with repeated measures was conducted on RT data with two within-subject factors and one between-subjects factor: Emotion (Angry vs. Happy) indicates the emotion of the target face; Crowd-size (3, 6, vs. 12) indicates the number of faces presented; Group (TD vs. ASD) indicates whether the participants were TD or ASD children. The results revealed a main effect of Emotion, $F(1,41)=18.00, p<.001, \eta_{p}^{2}=.305$, and more importantly, a significant interaction between Emotion and Group, $F(1,41)=$
5.505, $p=.024, \eta_{p}^{2}=.118$. No other main effects or significant interactions were observed. Subsequent analysis (Bonferroni correction) revealed that TD children detected angry targets faster than happy targets, $F(1,41)=23.33, p<.001, \eta_{p}^{2}=.363$, clearly indicating the Anger Superiority Effect. In contrast, although ASD children showed slightly faster mean RT for detecting angry targets compared to happy targets, the difference was not significant, $F(1$, $41)=1.680, p=.202, \eta_{p}^{2}=.039$ (Figure 1A).

Accuracy data were analyzed with a GLM analysis with the same factors as above. The results revealed a significant main effect of Emotion, $F(1,41)=5.977, p=.019, \eta_{p}^{2}=.127$ : accuracy was higher when the target emotion was angry than when it was happy (Figure 1B). There were no other main effects or interactions.

We also conducted the same analyses, with age, AQ and full-scale IQ as covariates. Although we found a significant effect of age, $F(1$, $40)=12.82, p=.001, \eta_{p}^{2}=.243$, indicating slower overall RTs in younger children, no other effects or interactions were found.

Line Task. A GLM analysis with repeated measures was conducted on the RT data with Target (Vertical vs. Tilted), and Crowd-size (3, 6, vs. 12), as within-subjects factors, and Group (ASD vs. TD) as the between-subjects factor. There were main effects of Target, $F(1,41)=$ 124.7, $p<.001, \eta_{p}^{2}=.753$, and Crowd-size, $F(2,82)=24.40, p<.001$, $\eta_{p}^{2}=.373$, and a significant interaction between Target and Crowdsize, $F(2,82)=37.55, p<.001, \eta_{p}^{2}=.478$. This indicated that RT to detect a vertical target among tilted lines increased with increasing crowd-size (implying serial search), whereas RT to detect a tilted line among vertical lines was almost constant regardless of crowd-size (implying parallel search; Figure 2). Importantly, there was no significant main effect of Group $\left(F(1,41)=.061, p=.806, \eta_{p}^{2}=\right.$ $.001)$ and no significant interactions involving Group (Target $\times$ Group: $F(1,41)=.190, p=.665, \eta_{p}^{2}=.005$; Crowd-size $\times$ Group: $F(2,82)=1.642, p=.200, \eta_{p}^{2}=.039 ;$ Target $\times$ Crowd-size $\times$ Group: $\left.F(2,82)=.742, p=.480, \eta_{p}^{2}=.018\right)$.

Accuracy data were also analyzed with a GLM analysis with the same factors. The results revealed a significant main effect of Crowdsize, $F(2,82)=29.50, p<.001, \eta_{p}^{2}=.418$ : accuracy at the crowd size of 3 was lower than at the other ( 6 and 12) crowd sizes $(p<.001, p<$ .001 , respectively), but accuracy was not significantly different when compared between the crowd sizes of 6 and of $12(p=.723)$. Again, there was no significant main effect of $\operatorname{Group}(F(1,41)=.114, p=$ $\left..738, \eta_{p}^{2}=.003\right)$ and no significant interactions involving Group $\left(\right.$ Target $\times$ Group: $F(1,41)=.001, p=.976, \eta_{p}^{2}<.001 ;$ Crowd-size $\times$ Group: $F(2,82)=1.09, p=.341, \eta_{p}^{2}=.026 ;$ Target $\times$ Crowd-size $\times$ Group: $\left.F(2,82)=2.42, p=.095, \eta_{p}^{2}=.056\right)$.

\section{Discussion}

The current study showed that angry schematic faces were detected more quickly than happy schematic faces (i.e., ASE) by typically developing children. This result is consistent with previous studies of adults ${ }^{1,10,11,22}$ and children ${ }^{22}$. More importantly, we did not observe ASE in children with ASD. This group failed to show significantly faster detection of angry faces compared to happy faces. Our results suggest that ASD children, in contrast to TD children, are not predisposed to allocate their attention quickly to angry faces. As they showed similar overall response times and accuracy as TD children, ASD children are clearly competent at the facial visual search task; however, they showed no effect of emotion, which suggests an absence of processing the emotions depicted on the faces. In addition, we tested the same participants on another visual search task, requiring detection of one line at a different angle from the others; there was no difference between the ASD and the TD groups on this control task. This result further confirmed that the group difference in the face task was not due to a different level of understanding of the task, or differing concentration on the task between ASD and TD. Although previous studies have shown superior performance on 


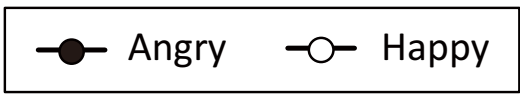

(A)
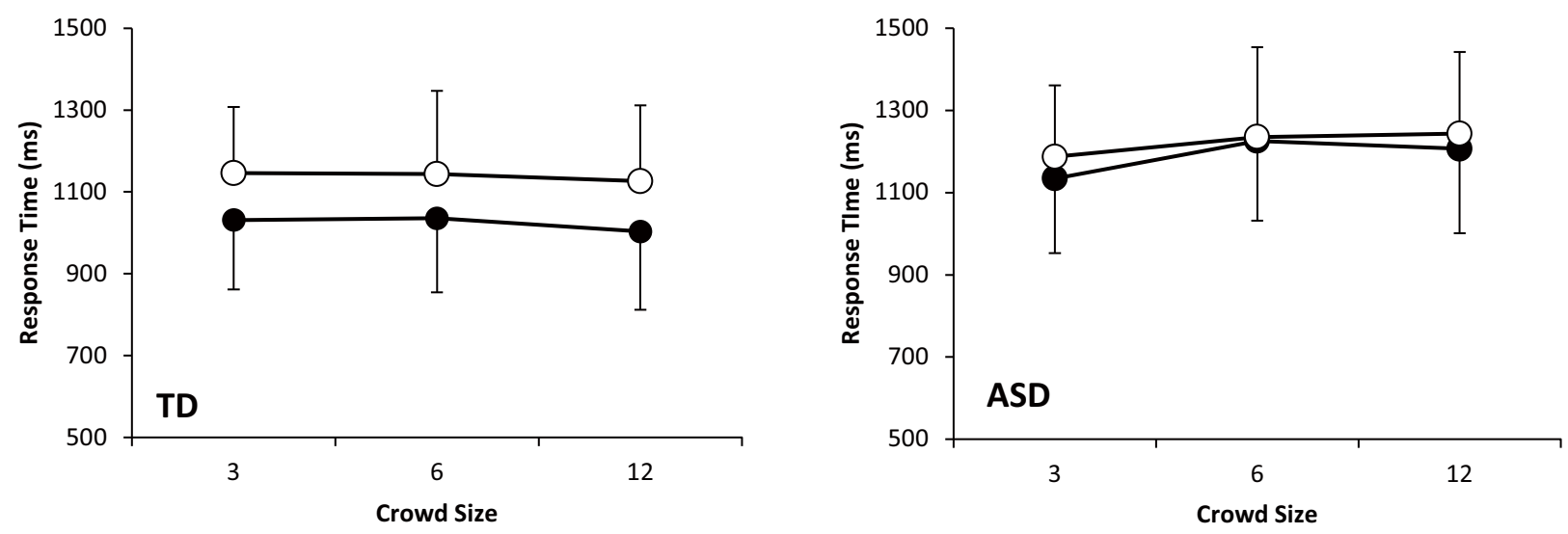

(B)
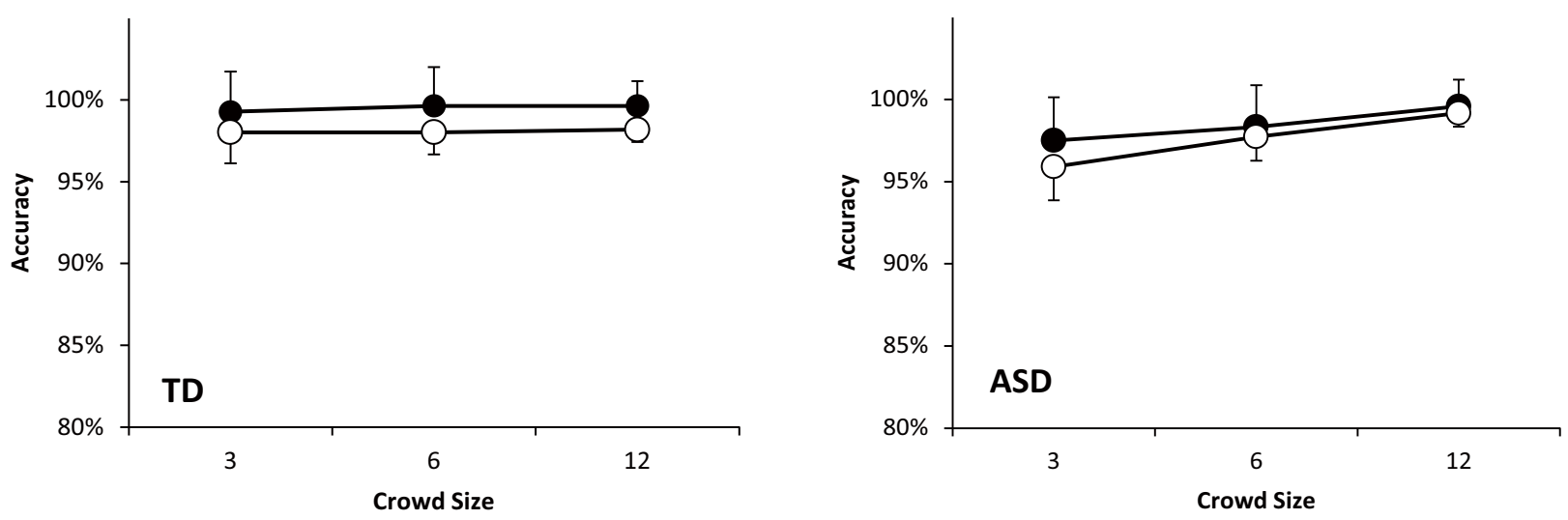

Figure 1 (A) Mean response times to detect angry and happy targets in the TD group (left) and the ASD group (right). Error bars indicate 95\% CI. (B) Mean accuracy for detection of angry and happy targets in the TD group (left) and the ASD group (right). Error bars indicate 95\% CI.

visual search tasks with non-social stimuli in $\mathrm{ASD}^{27-29}$, group differences were not observed when tasks were easy, resulting in a ceiling effect $^{27}$. In our study, although ASD children performed similarly to TD children in the line task, which may have resulted from a ceiling effect, the face task with the same procedure and same participants revealed a group difference. These results suggest that children with ASD showed a particular atypicality on the performance of the visual search task with facial emotional stimuli.

Sensitivity to different facial expressions normally emerges early in development. Infants in the first few months of life can discriminate between different emotions such as angry and happy ${ }^{32,33}$, and angry faces in particular capture infants' attention ${ }^{34}$. Five-year-old children already show ASE in the visual search paradigm ${ }^{22}$. These results are consistent with the idea that the detection of threat is an evolved and adaptive mechanism that is 'hard-wired' in humans to make them biologically prepared ${ }^{1,2}$. Thus, it is not surprising that typically developing 7-10-year-old children in the current study showed intact ASE, as we had hypothesized. In contrast, ASD children of the same age did not show ASE. Despite good performance in the odd item search, they did not appear to process the emotions on the stimulus faces. This result raises the possibility that predispositional mechanisms of quick threat detection presented in facial expressions, are not preserved in young children with ASD. However, some previous studies have shown ASE in individuals with $\mathrm{ASD}^{13-15}$, although the effect is less robust than in TD individuals ${ }^{13,14}$. Combining results of the current study and previous studies, we propose the following hypothesis: individuals with ASD, in contrast to TD individuals, lack functional predispositional mechanisms for allocating attention rapidly towards angry faces. However, they may compensatorily acquire attentional sensitivity to angry faces during development. Supporting this hypothesis, a recent study provided preliminary evidence for different underlying cognitive mechanisms even though individuals with ASD showed similar ASE as TD individuals ${ }^{25}$.

We showed clearly that TD children detect angry faces faster than happy faces. However, unlike many previous studies showing that RTs increased with increasing number of distractors (i.e., crowdsize $)^{12}$, our results showed stable RTs across three crowd sizes. Furthermore, previous studies have shown variable search efficiency (i.e., search slope; calculated as increase in RT by increase of crowd size) between threatening/negative targets and happy/positive targets $^{12}$. Threatening targets are relatively less affected by the number of distractors than non-threatening targets. In our study, however, search slopes for detection of angry targets and happy targets were not very different. This divergence may be due to the methods we employed. In a recent experimental comparison of threat detection paradigms $s^{24}$, when participants were required to judge whether or 

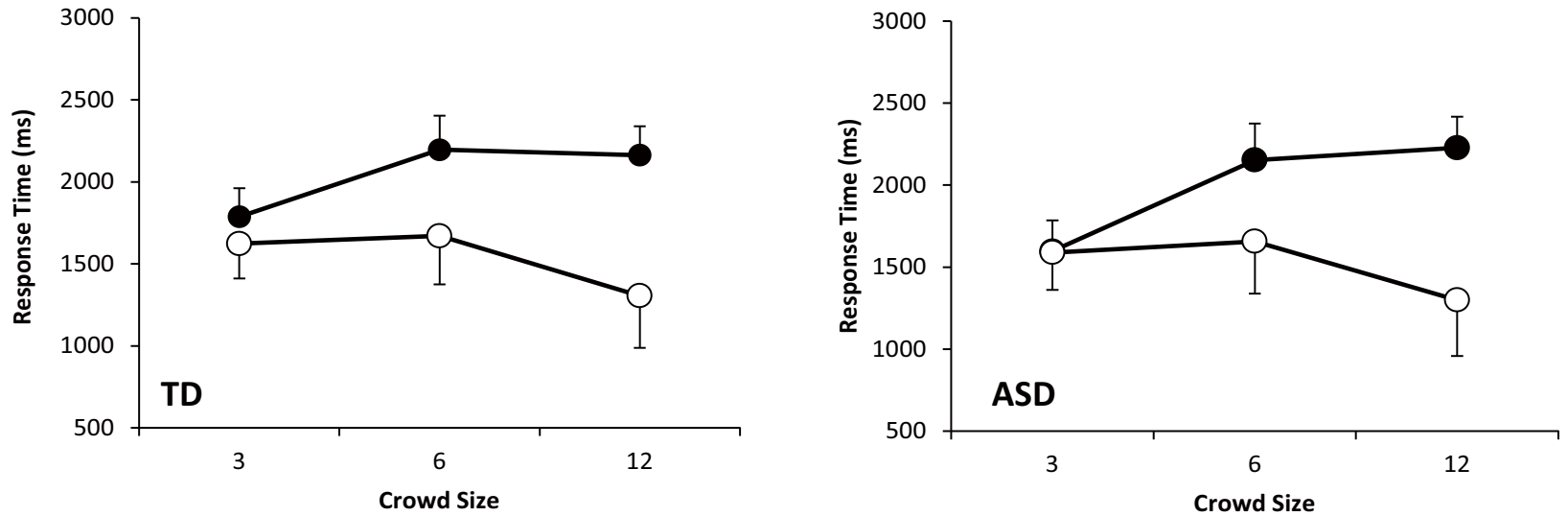

Figure $2 \mid$ Mean response times to detect vertical targets and tilted targets in the TD group (left) and the ASD group (right). Error bars indicate 95\% CI.

not a discrepant item was present in the matrix (classic visual search paradigm), search efficiency differed between threat-relevant and threat-irrelevant targets: only threat-irrelevant targets were affected by the number of distractors. In contrast, when participants knew that a target was present on the screen and they simply needed to find it, search efficiency across threat-relevant and threat-irrelevant targets was similar. The authors suggested that the latter procedure does not draw upon the "automaticity" of threat detection, but that it can still reveal advantageous attention capture by threatening stimuli ${ }^{24}$. In addition, as we used a relatively small stimulus presentation area, the distance between display items was small. Although our display was based on a previous study ${ }^{12}$, it may have emphasized the physical contrast between a target and distractors, resulting in stable RTs across crowd-sizes. However, our important finding remains: TD children clearly showed the advantage of threat detection, whereas ASD children did not.

Even though a face stimulus involved a much larger amount of physical information than a line, detection of a face generally took a shorter time in comparison with detection of a line. Humans may possess a visual system in which perceiving and responding to social stimuli is easier and faster than responding to non-social stimuli. Considering their relative speeds of detection of faces and lines, children with ASD also seemed to perceive the face stimulus as a face, not as a simple aggregation of geometric features.

Although many visual search studies have used schematic stimuli to eliminate low-level perceptual variations found in photographs of emotional expression, schematic stimuli have reduced ecological validity. It is therefore important to examine whether similar results would be obtained using photographs of faces. In particular, a display that does not include identical faces within the same matrix, could provide important evidence about the relationship between odd-item search performance and emotion processing in individuals with ASD.

In conclusion, the current study investigated attentional sensitivity to angry faces (Anger Superiority Effect) in 7-10-year-old children with and without ASD. Whereas TD children clearly showed faster detection of angry targets compared to happy targets, ASD children did not show such an effect. These results suggest that individuals with ASD, in contrast to TD individuals, lack the predispositional mechanisms for allocating attention towards angry faces. However, given previous reports of ASE in adults and relatively older children with $\mathrm{ASD}^{13-15}$, albeit less robust than in TD individuals ${ }^{13,14}$, the present results suggest that individuals with ASD may acquire attentional sensitivity to angry faces during development. Further investigation will be needed to test this possibility.

\section{Methods}

Ethics note. This study was carried out in accordance with the Guide for Experimentation with Humans by the Primate Research Institute, Kyoto University. The experimental protocol was approved by the Human Research Ethics Committee of the same institute (permit number, \#2010-30). The parents of all participants gave written informed consent to their child's participation in the study.

Participants. Twenty children with ASD ( 15 males and 5 females; mean age $=9.06$ years; $\mathrm{SD}=1.21$ years; range: 7 years 0 month -10 years 7 months) and 23 typically developing children (12 males and 11 females; mean age $=9.02$ years; $\mathrm{SD}=0.98$

Table 1 | Mean (SD) accuracy and response times for each group in the face and the line tasks

\begin{tabular}{|c|c|c|c|c|c|c|c|c|c|c|c|c|c|}
\hline & \multirow{3}{*}{$\begin{array}{c}\text { Target stimulus } \\
\text { Crowd size }\end{array}$} & \multicolumn{6}{|c|}{ Face task } & \multicolumn{6}{|c|}{ Line task } \\
\hline & & \multicolumn{3}{|c|}{ Angry } & \multicolumn{3}{|c|}{ Happy } & \multicolumn{3}{|c|}{ Vertical } & \multicolumn{3}{|c|}{ Tilted } \\
\hline & & 3 & 6 & 12 & 3 & 6 & 12 & 3 & 6 & 12 & 3 & 6 & 12 \\
\hline \multirow[t]{3}{*}{ TD } & Accuracy (\%) & 99.3 & 99.6 & 99.6 & 98.0 & 98.0 & 98.2 & 86.6 & 95.3 & 97.5 & 90.2 & 96.7 & 98.9 \\
\hline & & I & $1-7$ & (1.7) & (3. & 16 & 14 & (10.6) & (7. & 16 & 196 & (5.5) & 100 \\
\hline & $\begin{array}{c}\text { Response Time } \\
\text { (ms) }\end{array}$ & $\begin{array}{l}1031 \\
(400)\end{array}$ & $\begin{array}{l}1036 \\
(402)\end{array}$ & $\begin{array}{l}1003 \\
13091\end{array}$ & $\begin{array}{l}1146 \\
(373)\end{array}$ & $\begin{array}{l}1143 \\
(414)\end{array}$ & $\begin{array}{l}1126 \\
(407)\end{array}$ & $\begin{array}{l}1787 \\
(494)\end{array}$ & $\begin{array}{l}2129 \\
(644)\end{array}$ & $\begin{array}{l}2163 \\
(712)\end{array}$ & $\begin{array}{l}1623 \\
(409)\end{array}$ & $\begin{array}{l}1670 \\
(499)\end{array}$ & $\begin{array}{l}1305 \\
(346)\end{array}$ \\
\hline \multirow[t]{3}{*}{ ASD } & Accuracy (\%) & 97.5 & 98.3 & 99.6 & 95.9 & 97.7 & 99.2 & 89.6 & 95.4 & 92.5 & $\begin{array}{l}48.7 \\
88.7\end{array}$ & 96.3 & 98.8 \\
\hline & & (6.1) & (4.4) & (1.9) & (7.5) & (4.0) & $(2.6)$ & (15.7) & (13.1) & (18.3) & (14.6) & (5.7) & (3.0) \\
\hline & Response Time & 1134 & 1226 & 1206 & 1187 & 1235 & 1243 & 1597 & 2152 & 2227 & 1589 & 1657 & 1299 \\
\hline
\end{tabular}


Table 2 | Mean and SD of chronological age, WISC IQ scores, and AQ scores for the TD and ASD groups

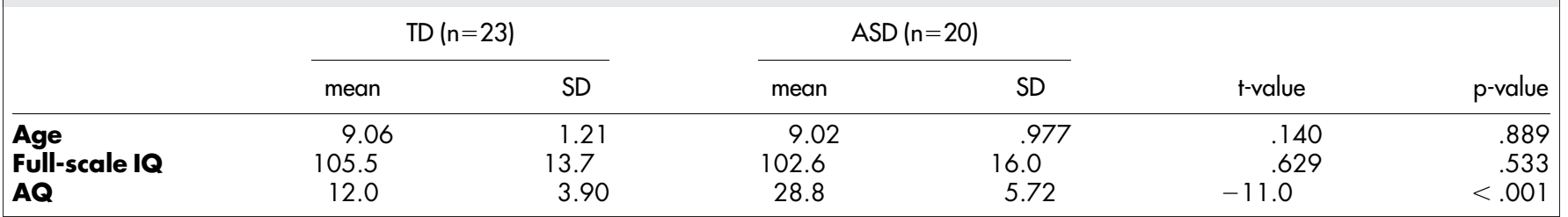

years; range: 7 years 1 month - 11 years 1 month) participated in this study. The ASD participants were diagnosed with Autism Spectrum Disorder, Asperger's syndrome, High-functioning Autism, Pervasive Developmental Disorder (PDD), or PDD-Not Otherwise Specified by child psychiatrists, based on either the DSM-IV or ICD10. They had been participating in the Developmental Disorders and Support for Acquiring Reading and Writing Skills project at the Kokoro Research Center, Kyoto University. Children with no history of any psychiatric condition were recruited via local schools and the community.

Intelligence Quotients (IQs) were measured using the Japanese version of the Wechsler Intelligence Scale for Children (either WISC-III or WISC-IV). The children's parents answered the Japanese version of the Autism Spectrum Quotient $(A Q)^{35}$. To be included in the TD group, participants had to meet the criterion of $A Q$ less than 20. Additionally, participants in both groups had to meet the criterion of IQ of 70 or higher. Additional 10 children were excluded from final sample because they did not meet the criteria of $\mathrm{AQ}$, IQ, or diagnosis.

Age, IQ scores, and AQ scores of both groups are listed in Table 1. Independent samples t-tests show that the groups were matched for age, $t(41)=.140, p=.889$, and full-scale IQ, $t(41)=.629, p=.533$. AQ scores showed a significant difference between the groups, $t(41)=-11.01, p<.001$

Apparatus. Visual stimuli were presented on a 15-inch touch-sensitive monitor with a resolution of 1024 by 768 pixels (Mitsubishi, RDT151TU), controlled by customwritten software in Visual Basic 2010 (Microsoft Corporation, Redmond, Washington, USA) running on a personal computer (HP Compaq 6730b/CT)

Stimuli. Each trial included presentation of a self-start key, a fixation picture, and face/ line stimuli. A light-blue-coloured rectangle, $179(\mathrm{~W}) \times 136(\mathrm{H})$ pixels $(5.3 \mathrm{~cm} \times$ $4.1 \mathrm{~cm} ; 7.6^{\circ} \times 5.9^{\circ}$ of visual angle), was used as the self-start key. It was presented at $1.5 \mathrm{~cm}\left(2.1^{\circ}\right.$ visual angle) from the bottom of the screen. In the middle of the rectangle, a trial number was presented so that participants could know how many trials they had completed. Fixation pictures were presented at the center of the screen and covering the whole stimulus area of faces. Twenty-four types of pictures of popular cartoon characters were used for the fixation pictures. The face stimuli were schematic pictures portraying angry, happy, and neutral facial expressions. The faces were drawn in black against a white background. All lines of faces including the outline, eyebrows, eyes, and mouth were drawn with lines of 2 pixels. The individual faces were 48 (W) by $54(\mathrm{H})$ (a)

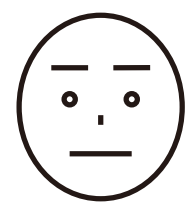

(b)

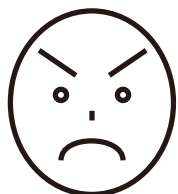

(c)

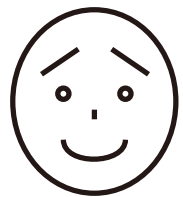

$(f)$

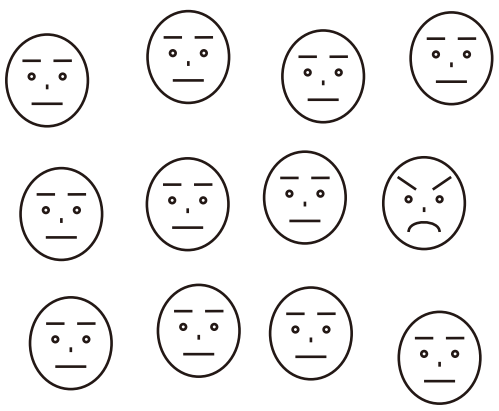

pixels $\left(1.4 \mathrm{~cm} \times 1.6 \mathrm{~cm}\right.$ on the screen; $2.0^{\circ} \times 2.3^{\circ}$ of visual angle; Figure $\left.3 \mathrm{~A} \sim \mathrm{C}\right)$. On each trial, 3,6 , or 12 face stimuli were presented inside a stimulus area of $268 \times 218$ pixels $\left(8.0 \mathrm{~cm} \times 6.5 \mathrm{~cm} ; 11.4^{\circ} \times 9.3^{\circ}\right.$ of visual angle) . The stimulus area was divided into $4 \times 3$ grids. We randomized the positions of face stimuli for each trial. First we randomly chose a grid for each face stimulus and then altered its position within a grid in a range of +-8 pixels from the center of the grid in both vertical and horizontal dimensions. This procedure resulted in a moderately irregular arrangement of the stimuli, intended to eliminate possible suprastimulus cues to the target's position ${ }^{36}$. Examples of stimulus displays are shown in Figure 3F, G.

The line stimuli consisted of two items that were distinguished from one another by a difference along a single dimension. The stimuli were all straight lines of $0.7 \mathrm{~cm}$ $\left(1.0^{\circ}\right.$ visual angle). One type of line was vertical, and the other was rotated $18^{\circ}$ counter-clockwise (Figure 3D, E). These stimuli were created based on a previous study ${ }^{27}$. They were presented in the same manner as the face stimuli described above.

Procedures. Face task. Participants were seated approximately $40 \mathrm{~cm}$ from the monitor with eye level at the center of the screen. They were first given 6 practice trials to help them understand the task. The practice session included every type of crowd size (3/6/12) and each emotion (angry/happy). At the start of the first trial of the practice session, participants were instructed to touch a discrepant object as quickly and accurately as possible. After it was ensured that participants understood the task, the experiment began. Each trial started when participants touched the self-start key, after which a picture of a popular character was presented for $500 \mathrm{msec}$ to keep the children's attention on the screen, and then the face stimuli were displayed. Face stimuli included one discrepant face,either an angry or a happy face, among $2 / 5 / 11$ neutral faces. The face stimuli were presented until a response was made. Correct responses were followed by a high-frequency auditory tone and a "correct" cartoon picture, whereas incorrect responses were followed by a low tone and an "incorrect" picture. Crowd size (3/6/12) and emotion type (angry/happy) were varied in a pseudorandom sequence within a session of 36 trials. Target position was also controlled by pseudorandom sequences. Each participant completed two 36-trial sessions, for a total of 72 trials.

Line task. After completing the face task, participants were given the line stimuli visual search task. The procedure was the same as for the face task, except that only two types of stimuli were used (vertical/tilted). Each participant completed two 36-trial sessions (d)

(e) (g)

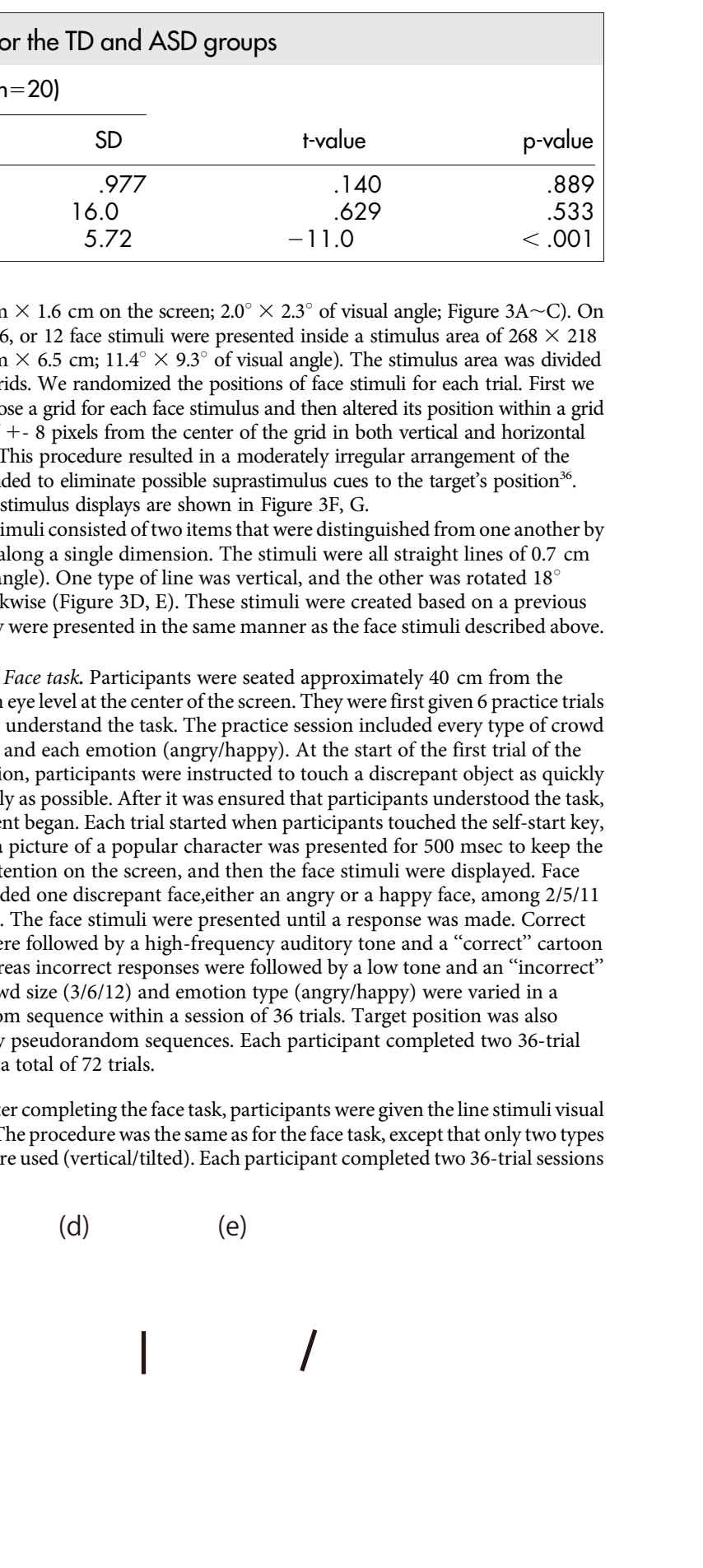

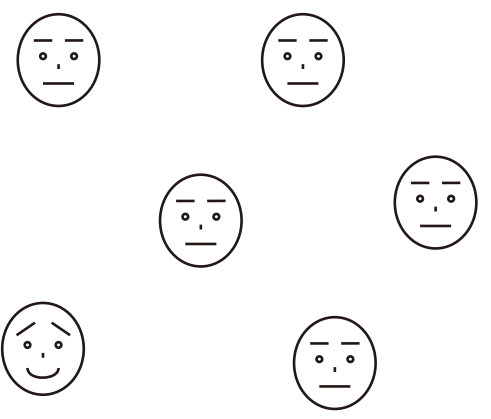

Figure 3 (A-E) Stimuli used in the study; (A) Neutral face, (B) Angry face, (C) Happy face in the face task; (D) Vertical line, (E) Tilted line in the line task. (F-G) Examples of displays in the face task; (F) One angry face and 11 distractor faces, (G) One happy face and 5 distractor faces. 
for a total of 72 trials. As with the face task, crowd size (3/6/12) and target type (vertical/tilted) were varied in a pseudorandom sequence.

1. Ohman, A., Lundqvist, D. \& Esteves, F. The face in the crowd revisited: A threat advantage with schematic stimuli. J. Pers. Soc. Psychol. 80, 381-396; DOI:10.1037//0022-3514.80.3.381 (2001).

2. Ohman, A. \& Soares, J. J. F. On the Automatic Nature of Phobic Fear Conditioned Electrodermal Responses to Masked Fear-Relevant Stimuli. J. Abnorm. Psychol. 102, 121-132; DOI:10.1037/0021-843X.102.1.121 (1993).

3. Vuilleumier, P. \& Schwartz, S. Emotional facial expressions capture attention. Neurology 56, 153-158; DOI:10.1212/WNL.56.2.153 (2001).

4. Emery, N. J. \& Amaral, D. G. The role of the amygdala in primate social cognition. Cognitive neuroscience of emotion, Richard D. Lane, Lynn Nadel (ed.), 156-191 (Oxford University Press, Oxford, 2000).

5. Ohman, A. The rote of the amygdala in human fear: Automatic detection of threat Psychoneuroendocrino. 30, 953-958; DOI:10.1016/j.psyneuen.2005.03.019 (2005).

6. Pessoa, L. \& Adolphs, R. Emotion processing and the amygdala: from a 'low road' to 'many roads' of evaluating biological significance. Nat. Rev. Neurosci. 11, 773-783; DOI:10.1038/nrn2920 (2010).

7. Hansen, C. H. \& Hansen, R. D. Finding the Face in the Crowd - an Anger Superiority Effect. J. Pers. Soc. Psychol. 54, 917-924; DOI:10.1037/00223514.54.6.917 (1988).

8. Horstmann, G. \& Bauland, A. Search Asymmetries With Real Faces: Testing the Anger-Superiority Effect. Emotion 6, 193-207; DOI:10.1037/1528-3542.6.2.193 (2006).

9. Pinkham, A. E., Griffin, M., Baron, R., Sasson, N. J. \& Gur, R. C. The Face in the Crowd Effect: Anger Superiority When Using Real Faces and Multiple Identities. Emotion 10, 141-146; DOI:10.1037/A0017387 (2010).

10. Eastwood, J. D., Smilek, D. \& Merikle, P. M. Differential attentional guidance by unattended faces expressing positive and negative emotion. Percept. Psychophys. 63, 1004-1013; DOI:10.3758/Bf03194519 (2001).

11. Fox, E. et al. Facial expressions of emotion: Are angry faces detected more efficiently? Cognition Emotion 14, 61-92; DOI:10.1080/026999300378996 (2000).

12. Horstmann, G. Visual search for schematic affective faces: Stability and variability of search slopes with different instances. Cognition Emotion 23, 355-379; DOI:10.1080/02699930801976523 (2009).

13. Ashwin, C., Wheelwright, S. \& Baron-Cohen, S. Finding a face in the crowd: Testing the anger superiority effect in Asperger Syndrome. Brain Cognition 61, 78-95; DOI:10.1016/j.bandc.2005.12.008 (2006)

14. Krysko, K. M. \& Rutherford, M. D. A threat-detection advantage in those with autism spectrum disorders. Brain Cognition 69, 472-480; DOI:10.1016/ j.bandc.2008.10.002 (2009).

15. Rosset, D. et al. More than just another face in the crowd: Evidence for an angry superiority effect in children with and without autism. Res. Autism Spect. Dis. 5, 949-956; DOI:10.1016/j.rasd.2010.11.005 (2011).

16. American Psychiatric Association. Diagnostic and statistical manual of mental disorders, 5th ed. (American Psychiatric Publishing, Arlington,VA, 2013).

17. Dawson, G., Webb, S. J. \& McPartland, J. Understanding the nature of face processing impairment in autism: Insights from behavioral and electrophysiological studies. Dev. Neuropsychol. 27, 403-424; DOI:10.1207/ s15326942dn2703_6 (2005).

18. Ashwin, C., Baron-Cohen, S., Wheelwright, S., O’Riordan, M. \& Bullmore, E. T. Differential activation of the amygdala and the 'social brain' during fearful faceprocessing in Asperger Syndrome. Neuropsychologia 45, 2-14; DOI:10.1016/ j.neuropsychologia.2006.04.014 (2007).

19. Critchley, H. D. et al. The functional neuroanatomy of social behaviour - Changes in cerebral blood flow when people with autistic disorder process facial expressions. Brain 123, 2203-2212; DOI:10.1093/brain/123.11.2203 (2000).

20. Wagner, J. B., Hirsch, S. B., Vogel-Farley, V. K., Redcay, E. \& Nelson, C. A. EyeTracking, Autonomic, and Electrophysiological Correlates of Emotional Face Processing in Adolescents with Autism Spectrum Disorder. J. Autism Dev. Disord. 43, 188-199; DOI:10.1007/s10803-012-1565-1 (2012).

21. Rozga, A., King, T. Z., Vuduc, R. W. \& Robins, D. L. Undifferentiated facial electromyography responses to dynamic, audio-visual emotion displays in individuals with autism spectrum disorders. Developmental Sci. 16, 499-514, DOI:10.1111/Desc.12062 (2013).

22. LoBue, V. More than just another face in the crowd: superior detection of threatening facial expressions in children and adults. Developmental Sci. 12, 305-313; DOI:10.1111/j.1467-7687.2008.00767.x (2009).

23. LoBue, V. \& DeLoache, J. S. Detecting the snake in the grass - Attention to fearrelevant stimuli by adults and young children. Psychol. Sci. 19, 284-289; DOI:10.1111/j.1467-9280.2008.02081.x (2008).

24. LoBue, V. \& Matthews, K. The snake in the grass revisited: an experimental comparison of threat detection paradigms. Cognition Emotion 28, 22-35; DOI:10.1080/02699931.2013.790783 (2014).
25. Isomura, T., Ogawa, S., Yamada, S., Shibasaki, M. \& Masataka, N. Preliminary evidence that different mechanisms underlie the anger superiority effect in children with and without Autism Spectrum Disorders. Front. Psychol. 5, 461; DOI:10.3389/fpsyg.2014.00461 (2014).

26. Isomura, T., Ogawa, S., Yamada, S., Shibasaki, M. \& Masataka, N. The Effect of Inversion on the Anger Superiority Effect in Children with and without Autism Spectrum Disorders. J. Psychol. Abnorm. Child. 3, 117; DOI:10.4172/23299525.1000117 (2014)

27. O’Riordan, M. A., Plaisted, K. C., Driver, J. \& Baron-Cohen, S. Superior visual search in autism. J. Exp. Psychol. Human 27, 719-730; DOI:10.1037//00961523.27.3.719 (2001)

28. Joseph, R. M., Keehn, B., Connolly, C., Wolfe, J. M. \& Horowitz, T. S. Why is visual search superior in autism spectrum disorder? Developmental Sci. 12, 1083-1096; DOI:10.1111/j.1467-7687.2009.00855.x (2009).

29. O'riordan, M. A. Superior visual search in adults with autism. Autism 8, 229-248; DOI:10.1177/1362361304045219 (2004)

30. Almeida, R. A., Dickinson, J. E., Maybery, M. T., Badcock, J. C. \& Badcock, D. R. Visual search performance in the autism spectrum II: The radial frequency search task with additional segmentation cues. Neuropsychologia 48, 4117-4124; DOI:10.1016/j.neuropsychologia.2010.10.009 (2010).

31. Almeida, R. A., Dickinson, J. E., Maybery, M. T., Badcock, J. C. \& Badcock, D. R. A new step towards understanding Embedded Figures Test performance in the autism spectrum: The radial frequency search task. Neuropsychologia $\mathbf{4 8}$ 374-381; DOI:10.1016/j.neuropsychologia.2009.09.024 (2010).

32. Barrera, M. E. \& Maurer, D. The perception of facial expressions by the threemonth-old. Child Dev. 52, 203-206; DOI:10.2307/1129231 (1981).

33. Bornstein, M. H. \& Arterberry, M. E. Recognition, discrimination and categorization of smiling by 5-month-old infants. Developmental Sci. 6, 585-599; DOI:10.1111/1467-7687.00314 (2003).

34. Serrano, J. M., Iglesias, J. \& Loeches, A. Visual-Discrimination and Recognition of Facial Expressions of Anger, Fear, and Surprise in 4-Month-Old to 6-Month-Old Infants. Dev. Psychobiol. 25, 411-425; DOI:10.1002/dev.420250603 (1992).

35. Wakabayashi, A., Baron-Cohen, S., Wheelwright, S. \& Tojo, Y. The AutismSpectrum Quotient (AQ) in Japan: a cross-cultural comparison. J. Autism Dev. Disord. 36, 263-270; DOI:10.1007/s10803-005-0061-2 (2006).

36. Duncan, J. \& Humphreys, G. W. Visual-Search and Stimulus Similarity. Psychol. Rev. 96, 433-458; DOI:10.1037/0033-295X.96.3.433 (1989).

\section{Acknowledgments}

This research was supported by a KAKENHI grant from the Japan Society for the Promotion of Science (JSPS; \#23653316 to NM); Grants-in-Aid for JSPS fellows (\#12J03878 to TI and \#09J02998 to HI); a grant from the Ministry of Education Culture, Sports, Science and Technology (MEXT) via its Global Centers of Excellence (gCOE) program (\#A06 to Kyoto University) and Grants for Excellent Graduate Schools, MEXT. This research was conducted as a part of a broader project on Developmental Disorders and Support for Acquiring Reading and Writing Skills headed by the Kokoro Research Center, Kyoto University, and we thank its director, Dr. Sakiko Yoshikawa, and members of the project for their support. We thank Dr. Ikuma Adachi and Dr. Hiroki Koda for valuable comments and suggestions about data analysis and manuscript improvement, Ms. Misako Ida for assistance in data collection, Dr. Masahiro Shibasaki for help in creating the task, and Dr. Elizabeth Nakajima and Dr. James R. Anderson for English revision and useful comments. We are grateful to all of the families that have kindly and generously given their time to participate in this study.

\section{Author contributions}

T.I. conceived the study, contributed to its design and coordination, and drafted the manuscript. T.I., H.I. and S.O. performed the experiments. N.M. supervised the project, data analysis, and editing of the manuscript. All authors participated in the interpretation of data, and reviewed the manuscript.

\section{Additional information}

Competing financial interests: The authors declare no competing financial interests.

How to cite this article: Isomura, T., Ito, H., Ogawa, S. \& Masataka, N. Absence of Predispositional Attentional Sensitivity to Angry Faces in Children with Autism Spectrum Disorders. Sci. Rep. 4, 7525; DOI:10.1038/srep07525 (2014).

This work is licensed under a Creative Commons Attribution-NonCommercialNoDerivs 4.0 International License. The images or other third party material in this article are included in the article's Creative Commons license, unless indicated otherwise in the credit line; if the material is not included under the Creative Commons license, users will need to obtain permission from the license holder in order to reproduce the material. To view a copy of this license, visit http:// creativecommons.org/licenses/by-nc-nd/4.0/ 